\title{
Prise En Charge Des Victimes Du Conflit Armé Du Nord Mali Dans Les Hôpitaux De Niamey
}

\author{
Chaibou MS, \\ Département d'Anesthésie-Réanimation- \\ Urgences-Hôpital National de Niamey - Niger
}

James Didier L,

Département de chirurgie - Hôpital National de Niamey

Daddy $\mathrm{H}$,

Salissou Abou A.N,

Département d'Anesthésie-Réanimation-Urgences-

Hôpital National de Niamey - Niger

Rhissa M,

Département de chirurgie - Hôpital National de Niamey

Gagara $M$,

Département d'Anesthésie-Réanimation-Urgences-

Hôpital National de Niamey - Niger

Garba I,

Département de chirurgie - Hôpital National de Niamey

Coulibaly $Y$,

Département Anesthésie-Réanimation Hôpital Point G - Mali

Sani $R$,

Département de chirurgie - Hôpital National de Niamey

doi: 10.19044/esj.2017.v13n18p204 URL:http://dx.doi.org/10.19044/esj.2017.v13n18p204

Abstract
Objectives: The aims of this study was to evaluate the management
of lesions in victims of the malian conflict patients admitted to Niamey
hospitals. Methods: A retrospective study was conducted in Niamey
hospitals from January $1^{\text {st }}, 2012$ to December, $31^{\text {th }}, 2013$. Data collected
included âge, sex, means of transport, topography of trauma, treatment and
outcome. Results: The sample included fifty one (51) patients. All patients
were male; the mean age was 29,5 years \pm 9 years, ranging from $17-71$ years.
The age between 26 and 35 were most affected in $47 \%$ of cases ( 24 patients),
soldiers represented $72.54 \%$ ( 37 patients); and civils $27.45 \%$ ( 14 patients).
$74.50 \%$ of our patients ( 38$)$ were been transported in Niamey by aircraft.
The trauma concerned the members in $54.90 \%$ ( 28 cases), canio facial in 
$17.60 \%$ (9 cases), abdomen in $11.80 \%$ (6 cases). We observed only one polytrauma. $66.66 \%$ (34) of patients were injured by firearms, $29.41 \%$ (15) by accidents of roads, 3,93\% by burn ( 2 patients). The lesions found were bone in $41.17 \%$ (21 patients), skin-muscle in $35.29 \%$ (18 patients), visceral in $9.80 \%$ (5 patients). Five patients died $(9.80 \%)$. Conclusion: the internationalization of conflicts requires a reorganization of the care by involving all the nearness health structures in order to ensure adequate care and neutrality.

Keywords: War wounds, firearm, Mali, Niamey hospitals

\section{Résumé}

Objectif : Evaluer la prise en charge des blessés victimes du conflit malien. Matériels et méthodes : Il s'agit d'une étude rétrospective et descriptive allant du $1^{\text {er }}$ janvier 2012 au 31 décembre 2013. Etaient inclus tous les patients victimes du conflit du nord Mali pris en charge dans les hôpitaux de Niamey. Les variables ci-après ont été étudiées : l'âge, le sexe, les moyens d'évacuation, la topographie des lésions, le traitement et l'évolution. Résultats : Nous avons enregistré 51 patients. Tous nos patients étaient des hommes, l'âge moyen était 29,5 ans \pm 9 ans avec des extrêmes de 17 à 71 ans. Les sujets ayant un âge compris entre 26 et 35 ans étaient les plus concernés avec 24 patients (47\%). Les militaires étaient les plus atteints avec $72,54 \%$; soit 37 patients et les civils $27,45 \%$ (14 patients). $74,50 \%$ de nos patients (38) ont été évacués à Niamey par voie aérienne. Les régions anatomiques lésées étaient les membres avec 28 cas $(54,90 \%)$, la région cranio-faciale avec neuf cas $(17,60 \%)$, six cas de traumatisme abdominal $(11,80 \%)$ ont été notifiés. Nous avons observé un seul polytraumatisé. Parmi nos patients trente-quatre $(66,66 \%)$ étaient blessés par balles; quinze (29,41\%) par accidents de la voie publique et deux par brulure $(3,93 \%)$. Les lésions rencontrées étaient osseuses dans 41,17\% (21 patients), cutanéomusculaires dans 35,29\% (18 patients) et viscérales dans $9,80 \%$ (5 patients). Cinq patients était décédés $(9,80 \%)$. Conclusion : L'internationalisation des conflits nécessite une réorganisation de la prise en charge en impliquant toute les structures sanitaires de proximité afin d'assurer des soins adaptés mais aussi la neutralité.

Mots-clés: Conflit armé, nord Mali, hôpitaux de Niamey

\section{Introduction}

Le déclenchement du conflit armé dans la bande sahélo saharienne et le déclenchement de l'opération "serval » a nécessité une réorganisation de la prise en charge des blessés au regard de la non disponibilité des 
structures hospitalières au nord Mali (abandonnées aux premières heures du conflit), de l'étendue de la zone de combat et pour des raisons de neutralité. C'est ainsi que les autorités et certaines Organisations Non Gouvernementales (ONG), en particulier le CICR ont mis en place un pont pour la prise en charge des victimes dans les structures hospitalières du Niger, ce qui a permis l'évacuation de nombreux blessés sur Niamey. Pour assurer une bonne compréhension de la pathologie traumatique de guerre des formations ont été effectuées par ces ONG. L'objectif de notre étude était d'évaluer la prise en charge des lésions chez les victimes du conflit malien évacuées au Niger (Bombert 2015, ICRC 2008, ICRC 2015).

\section{Matériels et méthode}

Il s'agit d'une étude rétrospective descriptive, allant du $1^{\mathrm{er}}$ janvier 2012 au 31 décembre 2013 ; soit deux ans. Etaient inclus tous les patients victimes du conflit du nord Mali évacués dans les hôpitaux de Niamey. Les variables suivantes ont été étudiées: âge, sexe, moyens d'évacuation, topographie des lésions, traitement et évolution. Les données ont été traitées à l'aide des logiciels épi info 2011, version 3.5.4 et Microsoft Word 2007.

\section{Résultats}

Cinquante-un (51) patients ont été pris en charge, tous de sexe masculin. L'âge moyen était de 29,50 ans \pm 9 ans avec des extrêmes allant de 17 ans à 71 ans. La tranche d'âge comprise entre 26 et 35 ans était la plus concernée avec 47\% (24 patients), suivie de la tranche 15-25 ans 33,40\% (17 patients). Un seul patient avait plus de 65 ans (1,96\%) (Tableau I).

Tableau I : Répartition des patients selon la tranche d'âge

\begin{tabular}{ccc}
\hline \hline Age (Année) & Effectif & Pourcentage (\%) \\
\hline \hline $15-25$ & 17 & 33,40 \\
$26-35$ & 24 & 47,00 \\
$36-65$ & 9 & 17,64 \\
$>65$ ans & 1 & 1,96 \\
Total & 51 & 100 \\
\hline
\end{tabular}

Les militaires représentaient 72,54\% (37 patients) et les civils-combattants 27,45\% (14 patients). Les moyens d'EVASAN (Evacuation Sanitaire) sur le Niger étaient aériens pour 38 blessés $(74,50 \%)$ et terrestres pour treize $(25,50 \%)$. Trente-quatre patients $(66,66 \%)$ étaient blessés par armes à feu, quinze $(29,41 \%)$ par accidents de la voie publique et deux $(3,93 \%)$ par brulures. (Figure $\mathbf{n}^{\circ} \mathbf{1}$ ) 


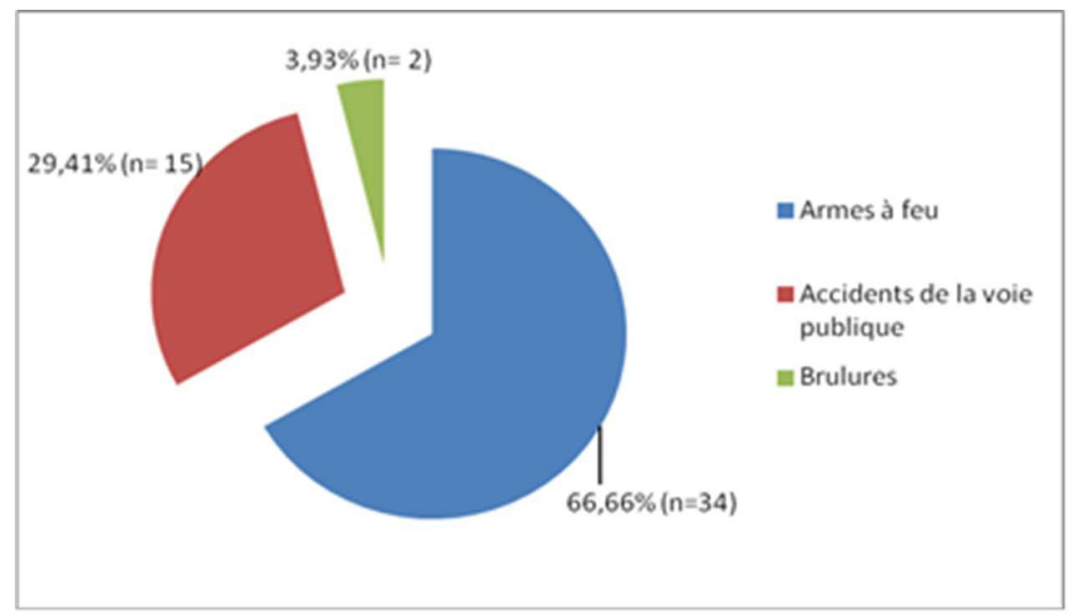

Figure $\mathbf{n}^{\circ} 1$ : Répartition des patients selon l'agent vulnérant

Les lésions concernaient les membres dans $54,90 \%$ des cas ; soit 28 patients (membres thoraciques: 51,51\%, membres pelviens : 48,49\%), la région cranio faciale dans $17,60 \% \quad$ (9 patients), l'abdomen dans $11,80 \%$ (6 patients). Les lésions étaient thoraciques et rachidiennes dans respectivement $7,80 \%$ (4 patients) et 5,90\% (3 patients). Nous avons observé un cas de polytraumatisme (Tableau II).

Tableau II : Siège du traumatisme

\begin{tabular}{ccc}
\hline \hline siège du traumatisme & Effectif & Pourcentage (\%) \\
\hline \hline Membres & & 54,90 \\
Cranio-facial & 28 & 17,60 \\
Abdomen & 9 & 11,80 \\
Thorax & 6 & 7,80 \\
Rachis & 4 & 5,90 \\
Polytraumatisme & 3 & 2,00 \\
Total & 1 & 100 \\
\end{tabular}

Les lésions rencontrées étaient osseuses chez 21 patients $(41,17 \%)$, cutanéomusculaires chez $18(35,29 \%)$ et viscérales chez 5 patients $(9,80 \%)$.(Figure $\mathbf{n}^{\circ}$ ) 


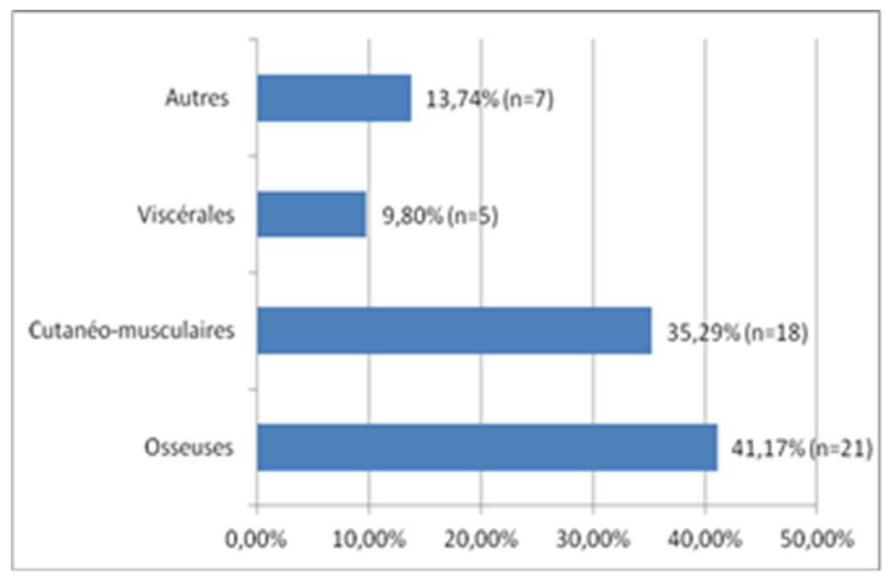

$\underline{\text { Figure } \mathbf{n}^{\circ} \mathbf{2}}$ : Types de lésion

La prise en charge initiale a débuté au Mali chez $11,76 \%$; soit 6 patients. $88,23 \%$ des patients ; soit 45 patients étaient des transferts directs sur Niamey. La structure de prise en charge à Niamey était l'Hôpital National de Niamey pour 37 blessés $(72,54 \%)$ et la clinique Alissa pour quatorze (27,45\%). La radiographie standard a été réalisée chez 26 patients $(50,98 \%)$, le scanner a été réalisé chez sept patients $(12,72 \%)$. Tous nos patients ont bénéficiés d'antalgiques ; 84,31\% d'antibiothérapie (43 patients). La sédation et l'intubation trachéale a été réalisées chez deux patients $(3,92 \%)$. Six patients $(11,76 \%)$ avaient été transfusés. Trente-trois patients $(64,70 \%)$ avaient bénéficié d'un geste sous anesthésie : anesthésie générale dans 57,57\% des cas (19 patients); rachianesthésie dans 30,30\% (10 patients) et anesthésie locale dans $12,13 \%$ (4 patients). L'ostéosynthèse était l'acte chirurgical le plus réalisé chez 27,45\% (14 patients) ; 11,76\% (6 patients) avaient bénéficié d'un parage. La laparotomie a été réalisée chez trois patients $(5,88 \%)$. Cinq patients était décédés $\quad(9,80 \%)$.

\section{Discussion}

Dans notre série la tranche d'âge comprise entre 26 et 35 ans représentait $47 \%$, ce résultat est supérieur à celui rapporté en Côte d' Ivoire (Konan 2011) qui avait trouvé $41 \%$ de sujets de 20 à 40 ans. L'âge moyen de nos patients était de 29,50 ans. D'autres auteurs avaient eu un âge moyen de 34,30 ans et 34 ans (Konan 2011, Kra 2003). En Afghanistan (Benois 2008) a trouvé un âge moyen de 28 ans. En effet ceci peut s'expliquer par le fait que les jeunes s'engagent facilement dans les conflits car les plus exposés au chômage et à la précarité. Tous nos patients étaient de sexe masculin, notre résultat est supérieur à ceux retrouvés par certains auteurs (Konan 2011; Bonnet 2015) avec respectivement 53,50\% et 57,50\%. Nous avons eu exclusivement des hommes par le fait qu'il s'agit des militaires du front et de 
combattants armés qui sont transférés pour des raisons de neutralités ; les femmes et les enfants étaient pris en charge dans les structures maliennes. Dans notre étude $72,54 \%$ de nos patients étaient des militaires, notre résultat est inférieur à celui de Hoffman en Afghanistan (Hoffman 2013) qui a trouvé 95,50\%. Ceci peut s'expliquer par le fait qu'après l'assaut plusieurs militaires non maliens ont été référés sur Niamey avant leur rapatriement sur leur pays d'origine. A cela s'ajoute les civils combattants qui étaient aussi systématiquement transférés à Niamey pour des raisons de sécurité et de neutralité humanitaire. Le rôle de l'évacuation hors du théâtre de combat a été noté par Martinez JY (Martinez JY 2009). Les traumatismes des membres étaient plus fréquents $(54,90 \%)$, ceci est inférieur à celui trouvé par Hoffman qui a trouvé un taux de 67\%, mais supérieur au 9\% de la Côte d' Ivoire (Hoffman 2013, Konan 2011). Les membres thoraciques étaient atteints dans $51,51 \%$ et les membres pelviens dans $48,49 \%$, nos résultats concordent à ceux retrouvés par la littérature (Donnat 2009, Bonnet 2015) avec $49,90 \%$ pour les membres thoraciques et $50,10 \%$ pour les membres pelviens. Dans notre étude $66,66 \%$ des patients ont été atteints par balles et $29,41 \%$ par les accidents de la voie publique, Konan et Kra ont eu respectivement $30,20 \%$ et $31,30 \%$ pour les armes à feu (Konan 2011, Kra 2003). En Afghanistan les atteintes par balles représentaient 28\% (Hoffman 2013). Pour les accidents de la circulation nos résultats sont inférieurs à ceux retrouvés dans la littérature avec respectivement 37,30\% et 53,40\% (Konan 2011, Kra 2003). En période de guerre les accidents surviennent par excès de vitesse dans des zones ou le réseau routier est quasi inexistant avec un risque de dérapage important. La mortalité était de 9,80\% ; elle était de 14\% et $13,30 \%$ en Afghanistan (Benois 2008, Hoffman 2013). Le taux de décès était de 45,70\% pour les pathologies chirurgicales en Côte d' Ivoire (Konan 2011).

\section{Conclusion}

La prise en charge des blessés de guerre évolue vue l'internationalisation des conflits avec comme conséquence la diversification et la délocalisation des unités de prise en charge afin de permettre la stricte neutralité dans les soins. Cette expérience doit être vulgarisée dans les zones de conflits dont la fonctionnalité des structures sanitaires n'est pas garantie.

\section{Conflits d'intérêts}

Les auteurs ne déclarent pas de conflit d'intérêt concernant les données présentées dans cet article 


\section{References:}

1. Bombert C, Chaufer M, Boissier J et al. 2015 Opération «serval » intérêt du «patient evacuation cordination cell». Médecine et armées ; 43 ; 4 :325-329.

2. ICRC 2008. Comment le «conflit armé » est-il défini en droit international humanitaire? Comité International de la Croix Rouge(CICR): prise de position, mars 2008 ICRC. 2015. War surgery Working With limited resources in armed conflict and other situations of violence. Volume 1, ICRC, $353 \mathrm{p}$

3. Konan K.J, Soro L., Koffi M., et al. 2012 Prise en charge des traumatisés aux urgences du CHU de Yopougon pendant la crise postélectorale. Rev Afr Anesth Med Urgence. ; 17 : 25-28

4. Kra O., Ehui E., Eholie S., et al. 2003 Morbidité en période d'aprèsguerre à l'hôpital de l'ECOMOG de Monrovia (Libéria). Méd Afr Noire ; 50 : 3416-3453.

5. Benois A, Gentile A, Fritsch N et al. 2009 Prise en charge de blessés de guerre: expérience d'une équipe médico-chirurgicale à kaboul. Réanoxyo, 3 ; 25 :73-75.

6. Donnat N, Clapson P., Debien B. 2009 Les bléssés des conflits actuels : épidémiologie des blessures de guerre. Réanoxyo ; $25 ; 2$ : 37-41.

7. Bonnet S., Bertani A., Savoie PH ; et al 2015. Humanitarian surgical care provided by a french forward surgical Team: ten years of providing medical Support to the Population of the Ivory Coast. Mil Med.;180(10):1075-82

8. Hoffman C. 2013 Bléssés de guerre français en Afghanistan de la blessure à la réinsertion: étude épidémiologique rétrospective d'une cohorte de 450 patients entre 2010 et 2012.Thèse de Doctorat en Médecine en France 2013.p 71. 122.

9. Martinez JY., Puidupin M.,Précloux P et al . 2009 Les enjeux de la prise en charge des bléssés actuels. Réanoxyo; 25; 2: 41- 46. 